\title{
RINGS WITH A FINITE SET OF NONNILPOTENTS
}

\author{
MOHAN S. PUTCHA \\ Department of Mathematics \\ N.C. State University \\ Raleigh, N.C. 27607 \\ and

\section{ADIL YAQUB} \\ Department of Mathematics \\ University of California \\ Santa Barbara, California 93106 \\ (Received October 16, 1978)
}

ABSTRACT. Let $R$ be a ring and let $N$ denote the set of nilpotent elements of $R$. Let $n$ be a nonnegative integer. The ring $R$ is called a $\theta_{n}$-ring if the number of elements in $R$ which are not in $N$ is at most $n$. The following theorem is proved: If $R$ is a $\theta_{n}$-ring, then $R$ is nil or $R$ is finite. Conversely, if $R$ is a nil ring or a finite ring, then $R$ is a $\theta_{n}$-ring for some $n$. The proof of this theorem uses the structure theory of rings, beginning with the division ring case, followed by the primitive ring case, and then the semisimple ring case. Finally, the general case is considered.

KEY WORDS AND PHRASES. Nil ring, Division ring, Primitive ring, Semisimple ring, Semigroup. 
1. INTRODUCTION.

Let $\mathrm{R}$ be a ring and let $\mathrm{N}$ denote the set of nilpotent elements of $\mathrm{R}$. Let $\mathrm{n}$ be a nonnegative integer. The ring $R$ is called a $\theta_{n}$-ring if the number of elements in $R$ which are not in $N$ is at most $n$; that is, $|R / N| \leq n$. One question which suggests itself is the following: what are necessary and sufficient conditions for a given ring $R$ to be a $\theta_{n}$-ring? The answer is given in the following.

\section{MAIN RESULTS.}

THEOREM 1. If $R$ is a $\theta_{n}$-ring, then $R$ is $n i l$ or $R$ is finite. Conversely, if $R$ is a nil ring or a finite ring, then $R$ is a $\theta_{n}$-ring for some integer $n$.

In the proof of Theorem 1, we use the structure theory of rings, beginning with the division ring case, followed by the primitive ring case, and then the semisimple ring case. Finally, we consider the general case.

In preparation for the proof of Theorem 1, we first prove the following lemmas.

LEMMA 1. Any subring and any homomorphic image of a $\theta_{n}$-ring is a $\theta_{n}$-ring.

This follows at once from the definition of a $\theta_{n}$-ring.

LEMMA 2. Let $R_{1}, R_{2}, \ldots, R_{n+1}$ be rings where each $R_{i}$ has an identity. Then the direct sum $R_{1}+R_{2}+\ldots+R_{n+1}$ is not a $\theta_{n}$-ring.

PROOF. Let $u_{1}=(1,0,0, \ldots, 0), u_{2}=(0,1,0, \ldots, 0), \ldots, u_{n+1}=(0,0, \ldots, 0,1)$, where each $u_{i}$ is an element of the direct sum $R_{1}+R_{2}+\ldots+R_{n+1}$. Then $u_{i} \quad$ for each $i=1,2, \ldots, n+1$, where $N$ denotes the set of nilpotent elements of $R$. Hence

$$
\left|\left\{R_{1} \dot{+} R_{2} \dot{+} \ldots \dot{+} R_{n+1}\right\} \backslash N\right|>n,
$$

and thus $R_{1} \dot{+} R_{2} \dot{+} \ldots \dot{+} R_{n+1}$ is not a $\theta_{n}$-ring.

LEMMA 3. If $D$ is a division ring which is also a $\theta_{n}$-ring, then $D$ is finite.

This follows at once from the difinition of a $\theta_{n}$-ring. 
LEMMA 4. If $R$ is a primitive ring which is also a $\theta_{n}$-ring, then $R$ is finite. In fact, $R$ is isomorphic to a complete matrix ring $D_{m}$ over a finite division ring $D$.

PROOF. Suppose $R \neq D_{m}$ for any division ring $D$ and any positive integer $\mathrm{m}$. (We shall show that this leads to a contradiction.) Then, by Jacobson's Density Theorem [1; p. 33], for each positive integer $q$, there exists a subring $R_{0}$ of $R$ which maps homomorphically onto $\mathrm{D}_{\mathrm{q}}$, for some division ring $\mathrm{D}$. In particular, there exists a subring $R_{1}$ of $R$ which maps homomorphically onto $D_{n+1}$. Hence, by Lemma 1 ,

$$
D_{n+1} \text { is a } \theta_{n} \text {-ring. }
$$

Now, define $A_{1}, A_{2}, \ldots, A_{n+1}$ to be the following $(n+1) \times(n+1)$ matrices in $D_{n+1}$ :

$A_{i}$ has zero entries in all rows except row $i$, but $A_{i}$ has entries of 1 throughout row $i$;

$$
(i=1,2, \ldots, n+1) \text {. }
$$

It is readily verified that each $A_{i}$ is idempotent and thus each $A_{i}$ is not nilpotent. Hence, $\left|D_{n+1} \backslash N\right|>n$, where $N$ denotes the set of nilpotent elements of $D_{n+1} \cdot$ Therefore, $D_{n+1}$ is not a $\theta_{n}$-ring, which contradicts (2.1). This contradiction shows that $\mathrm{R} \cong \mathrm{D}_{\mathrm{m}}$ for some division ring $\mathrm{D}$ and some positive integer $\mathrm{m}$. Now, since the division ring $D$ is a subring of the $\theta_{n}$-ring $D_{m}, D$ is finite, by Lemmas 1 and 3 . Thus, $R$ is finite, and the proof is complete.

LEMMA 5. If $R$ is a semisimple ring which is also a $\theta_{n}$-ring, then $R$ is finite.

PROOF. By contradiction. Thus, suppose that $R$ is a semisimple ring which is also a $\theta_{n}$-ring, and suppose $R$ is not finite. Since $R$ is semisimple, there exists ideal $I_{\alpha}(\alpha \in \Omega)$ of $R$ such that $[1 ; p .14]$

$$
\hat{n}_{\alpha \Omega} I_{\alpha}=(0) \text {; each } R / I_{\alpha} \text { is primitive. }
$$

By Lemma $1, R / I_{\alpha}$ is a $\theta_{n}$-ring. Hence, by (2.2) and Lemma 4 ,

$$
R / I_{\alpha} \cong D_{m}, R / I_{\alpha} \text { is finite, for any } \alpha \in \Omega \text {. }
$$


Now, choose $\alpha_{1} \in \Omega$, and having chosen $\alpha_{1}, \ldots, \alpha_{k}$ so that

$$
\sum_{i=1}^{k} \cdot R / I_{\alpha_{i}} \cong R / n_{i=1}^{k} I_{\alpha_{i}}
$$

choose $\alpha_{k+1} \in \Omega$ such that $n_{i=1}^{k} I_{\alpha_{i}} \notin I_{\alpha_{k+1}}$. That such $\alpha_{k+1}$ can always be so chosen is proved as follows: suppose no such $\alpha_{k+1}$ exists. Then $(0)=\prod_{\alpha \in \Omega} I_{\alpha}=\prod_{1=1} I_{\alpha_{1}}$, and hence (see $(2.4)$ )

$$
R \cong R / \bigcap_{i=1}^{k} I_{\alpha_{i}} \cong \sum_{i=1}^{k} R / I_{\alpha_{i}}
$$

Thus, using (2.3), we see that $R$ is finite, a contradiction. This contradiction shows that there exists $\alpha_{k+1} \in \Omega$ such that $n_{i=1}^{k} I_{\alpha_{i}} \nsubseteq I_{\alpha_{k+1}}$. Now, as we can see from (2.3), $R / I_{\alpha_{k+1}}$ is simple. Since, moreover, $\sum_{i=1}^{k} I_{\alpha_{i}} \notin I_{\alpha_{k+1}}$, we have $\bigcap_{i=1}^{k} I_{\alpha_{i}}+I_{\alpha+1}=R$. Hence, by applying the second isomorphism theorem, we readily verify that

$$
R / \bigcap_{i=1}^{k+1} I_{\alpha_{i}} \cong R / \bigcap_{i=1}^{k} I_{\alpha_{i}}+R / I_{\alpha_{k+1}} \cong \sum_{i=1}^{k+1} R / I_{\alpha_{i}}
$$

by $(2.4)$. In particular, we have

$$
\sum_{i=1}^{n+1} \cdot R / I_{\alpha_{i}} \approx R / n_{i=1}^{n+1} I_{\alpha_{i}} .
$$

$\mathrm{n}+1$

Hence, using Lemma $1, \sum_{i=1}^{*} R / I_{\alpha_{i}}$ is a $\theta_{n}$-ring. This, however, contradicts Lemma 2 (see (2.3)). This contradiction shows that $R$ is finite, and the lemma is proved.

We are now in a position to prove our main result, stated at the beginning.

PROOF of Theorem 1. Let $\mathrm{R}$ be a $\theta_{\mathrm{n}}$-ring, and let $\mathrm{J}$ denote the Jacobson radical of $R$. We claim that $J$ is nil. We prove this by contradiction. Thus, suppose $J$ is not nil. Then, for some $a, a \in J$, $a \notin N$, where $N$ denotes the set of nilpotent 
elements of $R$. Let

$$
S=\left\{a, a^{2}, a^{3}, \ldots, a^{n+1}\right\},(a \in J, a \notin N)
$$

Since $a \notin N, a^{i} \notin N$ for each $i=1,2, \ldots, n+1$, and hence $S \subseteq R \backslash N$. Therefore,

$$
|\mathrm{S}| \leq|\mathrm{R} \backslash \mathrm{N}| \leq \mathrm{n}
$$

since $R$ is $a \theta_{n}$-ring. In view of (2.5) and (2.6), we see that $a^{i}=a^{j}$ for some distinct positive integers $i, j$, and hence some power of a is idempotent, say $a^{k}$. Thus, $a^{k}$ is an idempotent element of $\mathrm{J}$, and hence, as is well known, $a^{k}=0$. Therefore, $a \in \mathrm{N}$, a contradiction. This contradiction proves that $\mathrm{J}$ is $\mathrm{nil}$.

Now, if $J=R$, then $R$ is nil. So suppose $J \neq R$. Then $R / J$ is a semisimple ring with more than one element. Moreover, by Lemma $1, \mathrm{R} / \mathrm{J}$ is a $\theta_{\mathrm{n}}$-ring, and hence by Lemma $5, \mathrm{R} / \mathrm{J}$ is finite. We have thus shown that $\mathrm{R} / \mathrm{J}$ is a finite semisimple ring with more than one element, and hence, as is well known, $\mathrm{R} / \mathrm{J}$ has an idenitity. Let $\overline{\mathrm{u}}$ be the identity element of $\mathrm{R} / \mathrm{J}$, and let

$$
N+u=\{n+u \mid n \in N\} ; N \text { is the set of nilpotents of } R \text {. }
$$

We claim that

$$
(\mathrm{N}+\mathrm{u}) \cap \mathrm{N}=\emptyset
$$

For, if $a \in(N+u) \cap N$, then $a \in N$ and $a=n+u$ for some $n \in N$. Hence $\bar{a}=\bar{n}+\bar{u}=$ an invertible element of $\mathrm{R} / \mathrm{J}$. Thus, $\bar{a}$ is an invertible element of $\mathrm{R} / \mathrm{J}$, which contradicts the hypothesis that $a \in N$ (and hence $\bar{a}$ is nilpotent). This contradiction proves (2.8). In view of (2.8), we have $N+u \subseteq R \backslash N$, and hence

$$
|N+u| \leq|R \backslash N| \leq n,
$$

since $R$ is a $\theta_{n}$-ring. But $|N|=|N+u|$, and hence by (2.9), $N$ is finite. The net result, then, is that both $N$ and $R \backslash N$ are finite, and hence $R$ is finite. We have thus shown that, in any case, $R$ is nil (if $J=R$ ) or $R$ is finite (if $J \neq R$ ). The converse part of Theorem 1 is trivial. 
We conclude with the following

REMARK. The analogue of Theorem 1 is not true for semigroups. To see this, let $\mathrm{N}$ be an infinite nil semigroup, and let $1 \notin \mathrm{N}$ be an identity element; that is, $1 \cdot x=x \cdot 1=x$ for all $x$ in $\mathrm{N}$, and $1 \cdot 1=1$

Let $R=N \cup\{1\}$. Then $R$ is a semigroup. Also, $|R \backslash N|=1$. But $R$ is neither nil nor finite.

\section{REFERENCE}

1. Jacobson, N. Structure of Rings, rev. ed., Amer. Math. Soc. Collog. Pub1., Vo1. 37, Providence, R.I., 1964. 


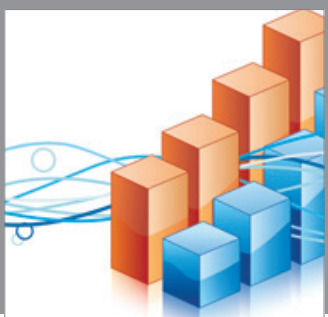

Advances in

Operations Research

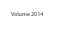

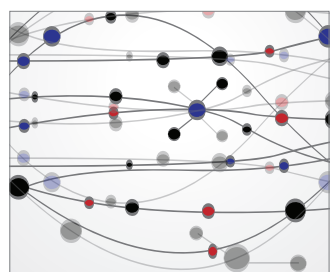

\section{The Scientific} World Journal
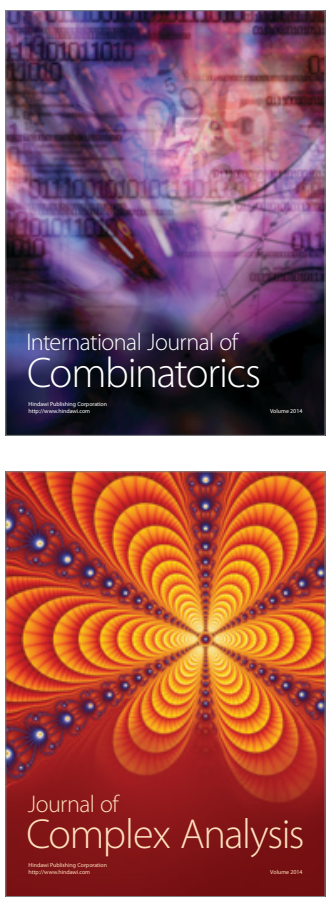

International Journal of

Mathematics and

Mathematical

Sciences
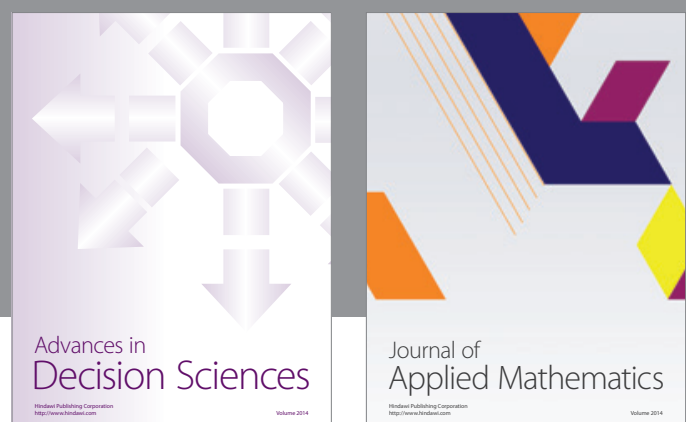

Journal of

Applied Mathematics
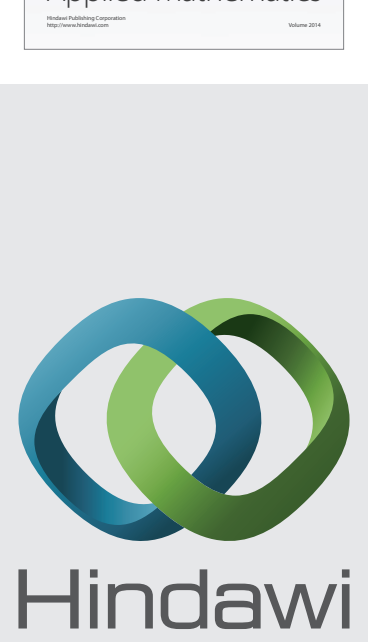

Submit your manuscripts at http://www.hindawi.com
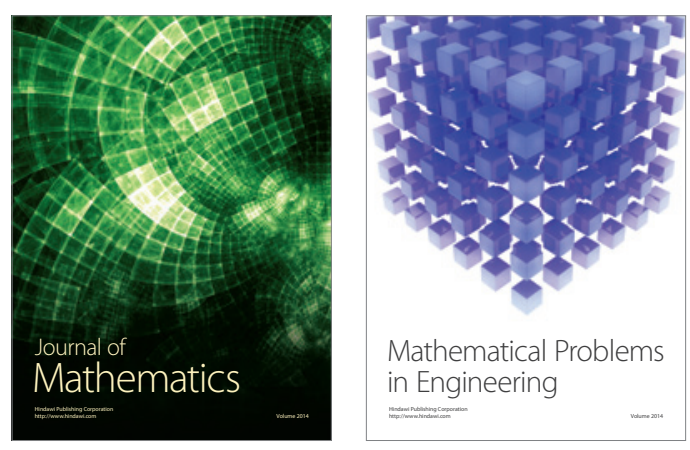

Mathematical Problems in Engineering
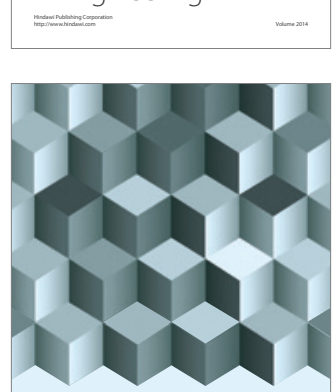

Journal of

Function Spaces
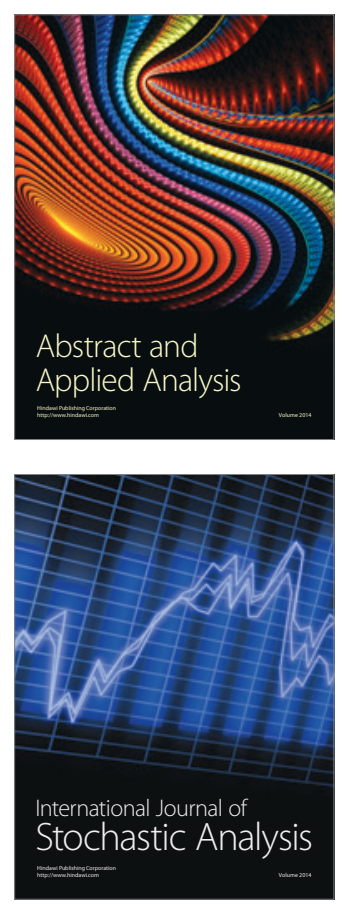

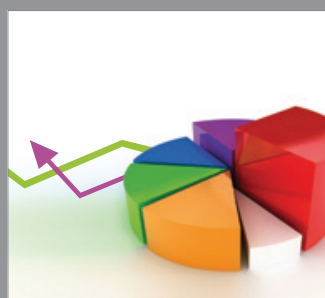

ournal of

Probability and Statistics

Promensencen
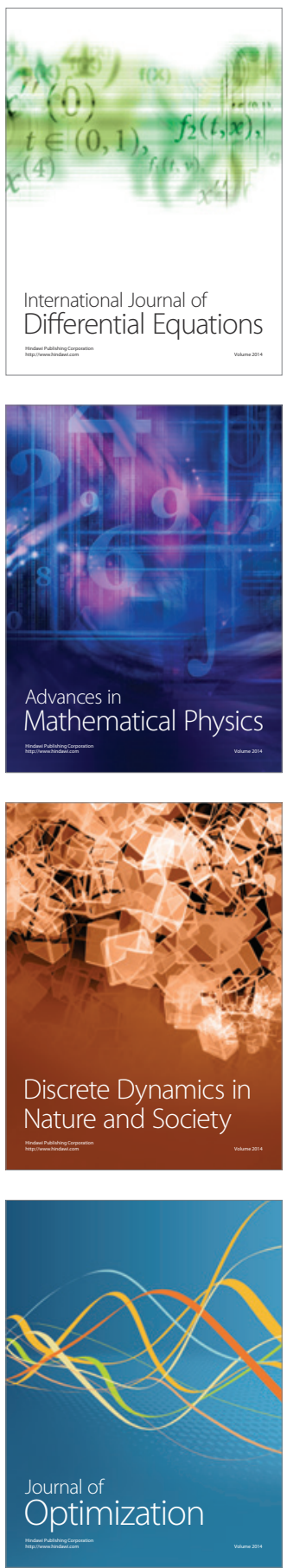